\title{
Genetic diversity of common Gasterophilus spp. from distinct habitats in China
}

Boru Zhang ${ }^{1,2+}$, Heqing Huang ${ }^{1 \dagger}$, Haoyu Wang ${ }^{1}$, Dong Zhang ${ }^{1}$, Hongjun Chu ${ }^{3}$, Xinping $\mathrm{Ma}^{4}$, Yan $\mathrm{Ge}^{3}$, Make Ente ${ }^{4}$ and Kai Li $i^{* *}$

\begin{abstract}
Background: Gasterophilus species are widely distributed around the world. The larvae of these flies parasitize the digestive tract of equids and cause damage, hindering horse breeding and protection of endangered species. However, study of the genetic structure of geographically distinct Gasterophilus populations is lacking. Here, we analyzed the genetic diversity of Gasterophilus pecorum, G. intestinalis, G. nasalis and G. nigricornis from three typical grasslands (meadow, desert and alpine steppes) in China as compared to published sequences from Italy, Poland and China (Daqing and Yili), based on the mitochondrial cytochrome c oxidase cox 1 and cox2 gene sequences.

Results: Haplotype diversity and nucleotide diversity of mitochondrial genes was generally high in all Gasterophilus populations. Due to the unique natural climatic conditions of the alpine steppe, there were high levels of genetic differentiation among different geographical populations of G. pecorum and G. nasalis, indicating that environmental variations influenced population genetic structure. Frequent exchanges between meadow and desert steppe Gasterophilus species resulted in low genetic differentiation. The highest exchange rates were found among $G$. intestinalis populations. Genetic differentiation was only observed on a large geographical scale, which was confirmed by analyzing population genetic structure. Three species, G. pecorum, G. intestinalis and G. nasalis, from meadow steppe showed a high emigration rate, indicating that the direction of Gasterophilus dispersal in China was from east to west.
\end{abstract}

Conclusions: Our results show that the four Gasterophilus species have a high level of genetic diversity and different degrees of genetic differentiation and gene flow among different populations of the same species, reflecting their potential to adapt to the environment and the environmental impact on genetic structure. Knowledge of the genetic structure, population history, and migration will help understand the occurrence and prevalence of gasterophilosis and provide a basis for controlling the local spread of Gasterophilus spp.

Keywords: Gasterophilus spp., Mitochondrial DNA, Genetic diversity, Population genetic structure

\section{Background}

Gasterophilus species are common obligate parasites in equines and are widely distributed worldwide. The genus Gasterophilus (Diptera: Oestridae) includes nine species [1]; of these, six are found in China: G. pecorum, G. intestinalis, G. nasalis, G. nigricornis, G. haemorrhoidalis and G. inermis [2-4]. Gasterophilus larvae parasitize the gastrointestinal tract of equids for 10-11 months and cause mucosal lesions,

\footnotetext{
* Correspondence: likai_sino@sina.com

${ }^{\dagger}$ Boru Zhang and Heqing Huang contributed equally to this work.

${ }^{1}$ Key Laboratory of Non-Invasive Research Technology for Endangered

Species, College of Nature Conservation, Beijing Forestry University, Beijing

100083, China

Full list of author information is available at the end of the article
}

gastrointestinal ulcers, peritonitis, anemia and gastric rupture, which can be severely debilitating $[5,6]$. The adults lay eggs directly on the hairs of host's body, except G. pecorum, which lays its eggs on grass [1, 7]. In European and American countries where animal husbandry is highly developed, the most prevalent species are $G$. intestinalis and G. nasalis, with the other Gasterophilus species rarely observed [6-13]. In traditional Chinese pastoral areas (i.e. Inner Mongolia, Xinjiang and Qinghai) the distribution of Gasterophilus larvae is more complicated than in other regions. For instance, Xilin Gol Grassland of Inner Mongolia Autonomous Region is an important pastoral area in China with an abundance of grass and water. Six species of Gasterophilus were found in Inner Mongolia, the 
predominant species being G. intestinalis followed by G. nasalis, which is consistent with the prevalence of Gasterophilus in other countries and regions [14]. The diversity index of Gasterophilus in this region was found to be 1.31 [14]. The same Gasterophilus species have been found in Xinjiang and Inner Mongolia, but G. pecorum was the predominant species (96.17\%) in Xinjiang, where the Gasterophilus species diversity index was very low (0.21) [14]. In Maduo County (MD) of Qinghai Province, only G. pecorum and G. nasalis have been documented, with the former being the predominant species $(93.71 \%)$ and contributing to a low diversity index (0.23) [14].

Mitochondrial DNA markers have been widely used in studies of insect taxonomy, population genetics and evolution. More specifically, mitochondrial cytochrome $c$ oxidase $\operatorname{cox} 1$ and $\operatorname{cox} 2$ genes have been used to examine inter- and intraspecific relationships in Diptera, Lepidoptera, Coleoptera, Hymenoptera and Hemiptera [15-19]. Previous investigations based on partial mitochondrial cox1 gene sequences revealed a high degree of genetic diversity that enabled differentiation of the Italian and Polish populations by PCR-restriction fragment length polymorphism [20]. In G. intestinalis from Daqing (DQ) and Yili (YL), China, genetic information is available for three mitochondrial genes, including cox1, NADH dehydrogenase subunit 5 gene (nad5) and $23 S$ ribosomal RNA gene [21]. However, little is known about the genetic structure of geographically distinct Gasterophilus populations. In the present study, we explored the population genetic structure and haplotype distribution patterns in several populations of G. pecorum, G. intestinalis, G. nasalis and G. nigricornis in China based on analyses of mitochondrial $\operatorname{cox} 1$ and $\operatorname{cox} 2$ gene sequences.

\section{Methods}

\section{Study sites and sample collection}

From February 2014 to December 2015, third-stage Gasterophilus larvae were collected in Kalamaili Nature Reserve (KNR) of Xinjiang Uigur Autonomous Region, Duolun County (DL) of Inner Mongolia Autonomous Region and MD of Qinghai Province (Additional file 1: Figure S1). A total of 97 G. pecorum and 63 G. nasalis specimens were obtained from the three locations, $42 \mathrm{G}$. intestinalis specimens were obtained from KNR and DL and 45 G. nigricornis specimens were obtained from KNR and DL (Additional file 2: Table S1). Larvae were preserved in ethanol and identified based on morphology [1].

Among the sampling sites in this study, KNR of Xinjiang Uigur Autonomous Region is located in the desert subregion of northwestern China with an altitude of $600-1464 \mathrm{~m}$, average annual temperature of $2.4{ }^{\circ} \mathrm{C}$, average summer temperature of $22{ }^{\circ} \mathrm{C}$, average annual precipitation of $159 \mathrm{~mm}$ and annual evaporation of 2090 $\mathrm{mm}$ [22]. DL is located at the southern end of Xilin Gol
Grassland in Inner Mongolia and is part of the Eurasian steppe; it has an elevation of 1150-1800 m, average annual temperature of $1.6{ }^{\circ} \mathrm{C}$, average summer temperature of $18.2{ }^{\circ} \mathrm{C}$, average annual precipitation of $385 \mathrm{~mm}$ and average annual evaporation of 1449.4-1672.8 $\mathrm{mm}$ [23]. Xinjiang and Inner Mongolia are zoogeographical regions belonging to the same district in China [24, 25]. MD is located in southwest Qinghai Province northwest of Guoluo Tibetan Autonomous Prefecture and has a plateau continental climate, the altitude is $4500-5000 \mathrm{~m}$, annual average temperature is $-4.1{ }^{\circ} \mathrm{C}$, average summer temperature is $<10{ }^{\circ} \mathrm{C}$, average annual precipitation is $303.9 \mathrm{~mm}$, and average annual evaporation is 1331.21 $\mathrm{mm}[26]$.

\section{DNA extraction, amplification and sequencing}

Total genomic DNA was extracted using a standard phenol/chloroform protocol [27], with minor modifications. Extracted DNA was frozen in DNase-free water and stored at $-20{ }^{\circ} \mathrm{C}$. A $632 \mathrm{bp}$ fragment of the mitochondrial cox1 gene was amplified with primers Gco1s (5'-CAG TTG GAA TAG ACG TTG ATA CTC-3') and Gcolan (5'-AGG AAG TTC AGA ATA GCA GTG TTC-3') [20], and a $688 \mathrm{bp}$ fragment of the mitochondrial cox 2 gene was amplified with primers TL2-J-3037 (5'-ATG GCA GAT TAG TGC AAT GG-3') and TK-N-3785 (5'-GTT TAA GAG ACC AGT ACT TG-3') [28]. PCR amplification of $\operatorname{cox} 1$ was performed according to a previously described protocol [20]. PCR conditions for cox 2 were as follows: $94{ }^{\circ} \mathrm{C}$ for $2 \mathrm{~min}, 35$ cycles of $94{ }^{\circ} \mathrm{C}$ for $30 \mathrm{~s}, 55^{\circ}$ $\mathrm{C}$ for $30 \mathrm{~s}$, and $72{ }^{\circ} \mathrm{C}$ for $1 \mathrm{~min}$, and $25{ }^{\circ} \mathrm{C}$ for $2 \mathrm{~min}$. PCR products were visualized by using $1 \%$ agarose gel electrophoresis, purified with the BigDye XTerminator Purification kit (Applied Biosystems, Foster City, CA, USA) and sequenced on an ABI 3730XL Genetic Analyzer (Applied Biosystems, Foster City, CA, USA). Sequences obtained were deposited in the GenBank database under the accession numbers MG815141-MG8 15634.

\section{Data analysis}

Sequences generated in this study as well as published sequences of G. intestinalis (GenBank: GU265738-GU265748, GU299281-GU299283 and KR230402-KR230415) and G. nasalis (GenBank: GU265749-GU265758 and GU299284GU299286) were used for data analysis. Raw sequences were proofread and edited using BioEdit v.7.0.9.0 [29]. Alignment was performed with the ClustalW algorithm in MEGA v.7.0 software [30], which was also used for basic sequence statistical analysis. Haplotype identification, haplotype diversity $(H d)$, and nucleotide diversity $(\pi)$ were estimated using DnaSP v.5.0 [31]. The Kimura two-parameter method was used to calculate genetic distances among populations in MEGA. DnaSP v.5.0 was used to evaluate population 
pairwise fixation index (Fst) statistics and gene flow $(\mathrm{Nm})$ values. Phylogenetic trees were constructed by the neighbor-joining (NJ) method using MEGA and were assessed with 1000 bootstrap replicates [32]. Haplotype networks were constructed using TCS v.1.21 [33] with a 95\% parsimony criterion. The Likelihood Analysis with Metropolis Algorithm using Random Coalescence (LAMARC) programme [34] was used to estimate population size $(\theta)$, exponential growth rate $(g)$ and migration rate based on the maximum-likelihood approach. The search strategy included three replications of 10 short initial chains and two long final chains. The initial chains were run with 500 samples and the sampling interval was set to 20 . The final chains were performed with 10,000 samples with the same sampling interval. A burn-in of 1000 samples was used for each chain.

\section{Results}

\section{Haplotype diversity}

A total of 247 cox 1 sequences were obtained for $G$. pecorum, G. intestinalis, G. nasalis and G. nigricornis, co-analyzed with 41 published sequences of $G$. intestinalis from China (DQ and YL), Italy and Poland, and of G. nasalis from Italy and Poland. An analysis of all cox 1 sequences identified a total of 154 haplotypes. The haplotype distribution, $H d$ and $\pi$ of each population based on the mitochondrial cox1 gene are shown in Additional file 2: Table S1. The values for $H d$ and $\pi$ were as follows: G. pecorum $(H d=0.976, \pi=0.01581) ; G$. intestinalis $(H d=0.983$, $\pi=0.01066)$; G. nasalis ( $H d=0.953, \pi=0.01226)$; and G. nigricornis ( $H d=0.973, \pi=0.01719)$. For each species, only two to five common haplotypes were observed within localities in China and there were no shared haplotypes across G. intestinalis and G. nasalis populations in Italy and Poland.

The 247 cox 2 sequences of the four Gasterophilus species defined 127 haplotypes. The haplotype distribution, $H d$ and $\pi$ of each regional population based on the mitochondrial cox2 gene are shown in Additional file 2: Table S2. The values for $H d$ and $\pi$ were as follows: $G$. pecorum ( $H d=0.01093, \pi=0.959)$; $G$. intestinalis $(H d=$ $0.00592, \pi=0.951) ; G$. nasalis ( $H d=0.01003, \pi=$ 0.961 ); and $G$. nigricornis ( $H d=0.01217, \pi=0.918$ ). Similar to cox1, only two to six common cox 2 haplotypes were observed within populations of each species.

\section{Haplotype networks}

\section{Gasterophilus pecorum}

The minimum spanning network calculated with TCS software using cox 1 haplotypes of G. pecorum had three sub-networks (Fig. 1a). Of the 48 cox 1 haplotypes, two (GpH4 and GpH19) were shared between KNR and DL and three (GpH1, GpH11 and GpH24) were shared between KNR and MD, while no common haplotypes were detected between DL and MD. The sub-networks formed a star-like structure that was derived from haplotype $\mathrm{GpH} 4$. Most unique haplotypes in the DL population were distributed around $\mathrm{GpH} 4$, whereas those unique to the MD population mostly surrounded GPH11. Haplotypes in the KNR population were more evenly distributed throughout the network. Individuals of $\mathrm{GpH} 37$ and $\mathrm{GpH} 48$ constituting a single sub-network were all from $\mathrm{MD}$. In general, the cox 2 haplotypes formed two sub-networks with $\mathrm{GpH} 17$ and $\mathrm{GpH} 43$ as the central haplotypes (Fig. 1b). The haplotype distribution of each geographical population was similar to that of cox1. Individuals in the GpH43-centric sub-network were the same as those in the cox 1 haplotype sub-network comprising only MD haplotypes. Of the 45 cox 2 haplotypes, three $(\mathrm{GpH} 2, \mathrm{GpH} 5$ and $\mathrm{GpH} 17)$ were shared between KNR and DL, four (GpH2, GpH3, GpH4 and GpH10) were shared between KNR and MD, and $\mathrm{GpH} 2$ was shared by all three populations.

\section{Gasterophilus intestinalis}

The minimum spanning network calculated with TCS software using cox 1 haplotypes of G. intestinalis had a single network and no sub-networks. Of the 48 cox1 haplotypes, three (GiH1, GiH4 and GiH13) were shared by KNR and DL, and one each was shared by KNR and DQ (GiH13), DL and DQ (GiH26), and DL and YL (GiH20). The network structure was dominated by a single haplotype (GiH4) (Fig. 2a). Haplotypes from Italy and Poland were all private haplotypes (i.e. found in only one population) and were less directly linked to those from China localities. The cox 2 haplotype network had a star-like structure, with the most abundant haplotype (GiH6) in the center and distributed across all geographical regions (KNR and DL) (Fig. 2b). The second most frequent haplotype GiH9 was also shared by KNR and DL.

\section{Gasterophilus nasalis}

The minimum spanning network calculated with TCS software using cox 1 haplotypes of G. nasalis had two sub-networks. Four (GnH2, GnH6, GnH7 and GnH17) of the 33 haplotypes were shared between KNR and DL, and two (GnH6 and GnH17) were shared among KNR, DL and MD (Fig. 3a). All haplotypes from Italy and Poland were private. Haplotypes in Poland constituted a separate sub-network, while those in Italy were less directly linked to haplotypes from localities in China. The cox2 haplotype network was dominated by GnH16, which was represented by a single individual (Fig. 3b). Haplotypes GnH5, GnH6 and GnH11 were shared between KNR and MD, while GnH27 was common to DL and MD. The MD haplotypes were mostly focused on branches centered on $\mathrm{GnH} 5$, there was no similar phenomenon in the cox1 haplotype network. Haplotypes in KNR and DL were randomly distributed throughout the network. 

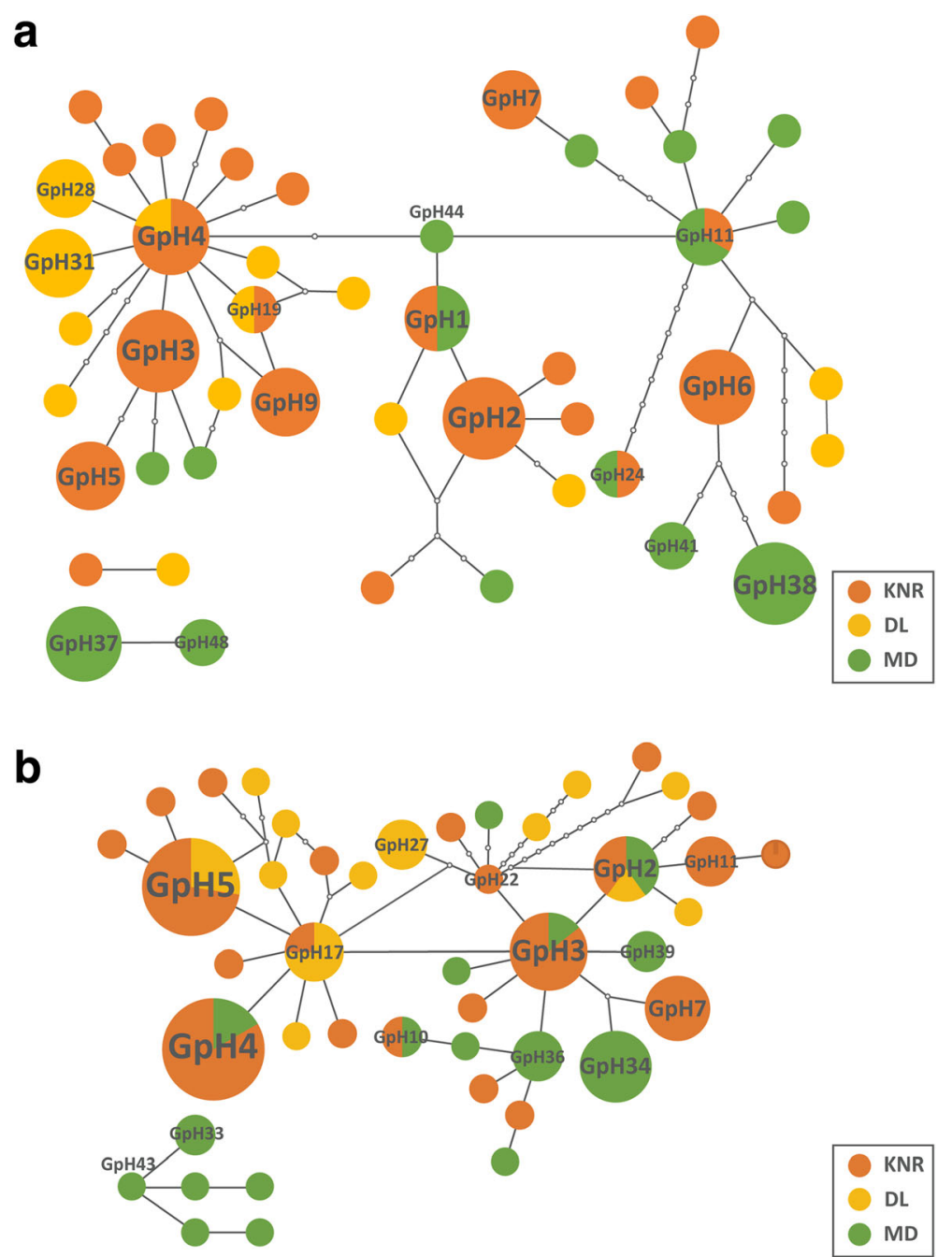

Fig. 1 Haplotype networks for G. pecorum. a Haplotype network for G. pecorum based on the cox 1 gene. b Haplotype network for G. pecorum based on the cox2 gene

\section{Gasterophilus nigricornis}

The minimum spanning network calculated with TCS software using cox1 haplotypes of G. nigricornis had two sub-networks centered around GniH6 and GniH26 (Fig. 4a). Most GL haplotypes were distributed around GniH26. The cox2 haplotype network comprised a single network centered around haplotype GniH16 (Fig. 4b). Five of the 22 haplotypes differed by more than eight mutations from haplotype GniH16, and most DL haplotypes were more closely related to the ancestral one.

\section{Phylogenetic analysis}

\section{Gasterophilus pecorum}

The phylogenetic tree constructed with the NJ method based on the cox 1 gene included 48 haplotypes divided into seven clades (Fig. 5a). Clade 1 included $73.68 \%$ of DL and $50 \%$ of KNR samples with seven and nine private haplotypes, respectively. Clades 3 and 7 comprised $77.42 \%$ of MD samples, and all haplotypes except GpH41 and GpH44 were private. An NJ tree constructed from cox2 sequences had five clades (Fig. 6a). DL haplotypes, including nine that were private, were mainly distributed in Clades 2 and 3, accounting for $84.21 \%$ of individuals in the DL population. Clades 1 and 5 contained the remaining 13 private haplotypes of MD except for GpH35, and included 87.10\% of individuals from MD. KNR haplotypes were mainly found in Clades 1 and 2, the former included seven private haplotypes and $40 \%$ of individuals, while the latter contained six private haplotypes and $50 \%$ of individuals.

\section{Gasterophilus intestinalis}

The NJ tree constructed based on the cox 1 gene included 44 haplotypes that were divided into four clades (Fig. 5b). Haplotypes from Italy and Poland were independently 

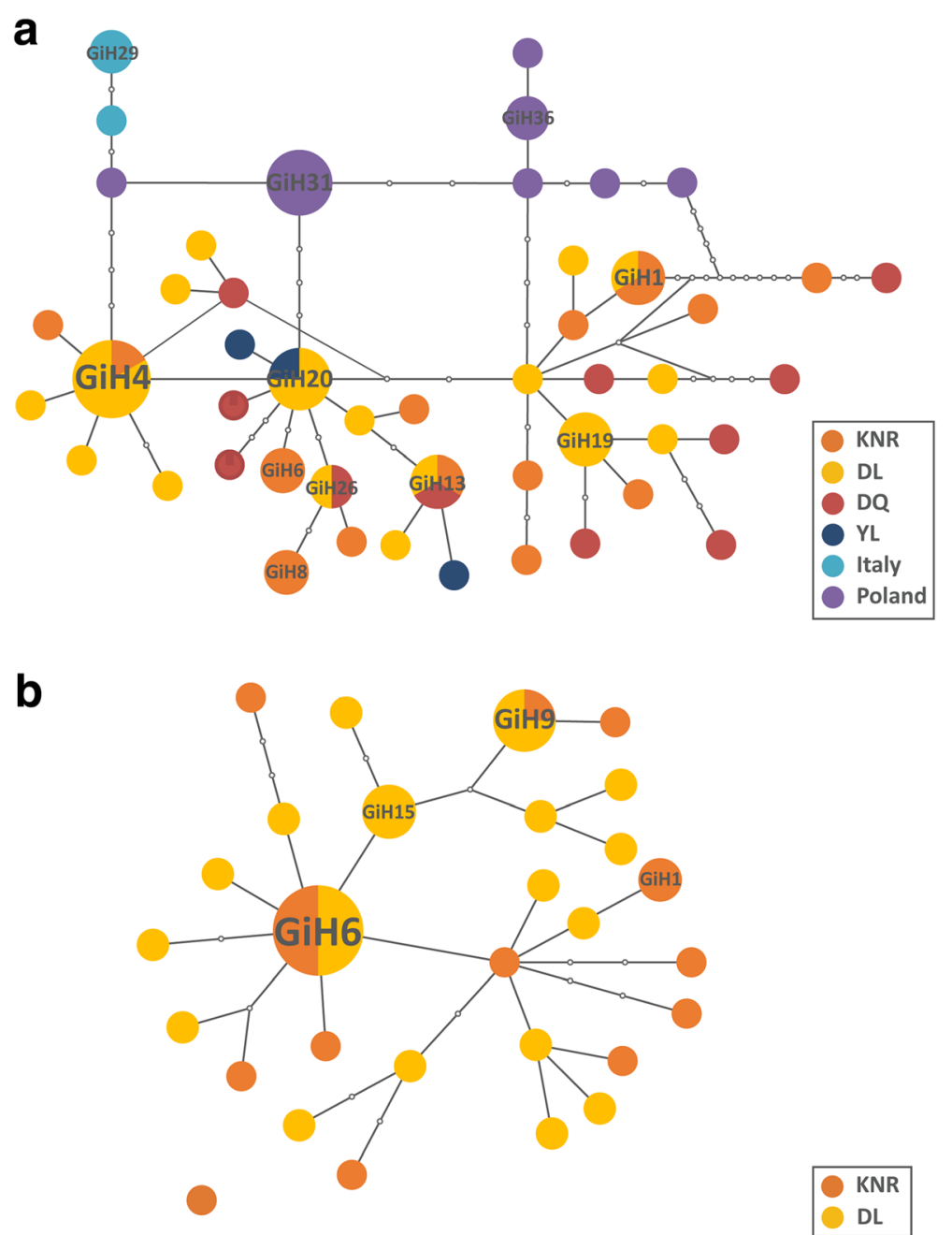

Fig. 2 Haplotype networks for G. intestinalis. a Haplotype network for G. intestinalis based on the cox 1 gene. b Haplotype network for G. intestinalis based on the cox2 gene

clustered and all were distributed in Clade 2, with the populations from the four geographical regions of China forming the three remaining clades. In the NJ tree constructed based on cox1 haplotypes, KNR and DL populations did not show any obvious clustering (Fig. 6b).

\section{Gasterophilus nasalis}

The NJ tree constructed based on cox 1 haplotypes had three clades (Fig. 5c). Three of the Chinese populations (KNR, DL and MD) formed Clade 1, and the Italian and Polish populations formed Clades 2 and 3, respectively. The NJ tree constructed based on the cox 2 gene had 31 haplotypes divided into three clades (Fig. 6c). The KNR population was mainly distributed in Clade 1, which included 10 private haplotypes and $66.67 \%$ of KNR individuals. All four private haplotypes of MD were distributed in clade 3, which included $77.78 \%$ of MD individuals. The DL population was distributed in three clades with no obvious aggregation.

\section{Gasterophilus nigricornis}

The NJ tree constructed from cox 1 haplotypes was divided into three clades (Fig. 5d). The DL population was mainly distributed in Clade 1, which contained eight private haplotypes and $56.25 \%$ of DL individuals, whereas the KNR population was evenly distributed. The NJ tree constructed based on cox 1 haplotypes was also divided into three clades (Fig. 6d). The private haplotypes of DL were all distributed in Clade 1, which included $68.75 \%$ of DL individuals. The haplogroup clustering of the KNR population was similar to that of cox1.

\section{Genetic structure and gene flow Gasterophilus pecorum}

Mean genetic distances between different populations were calculated based on cox 1 and $\operatorname{cox} 2$ sequences. Distances based on cox 1 gene sequences among DL, KNR and MD populations were 0.008 (KNR and DL), 0.023 (KNR and 

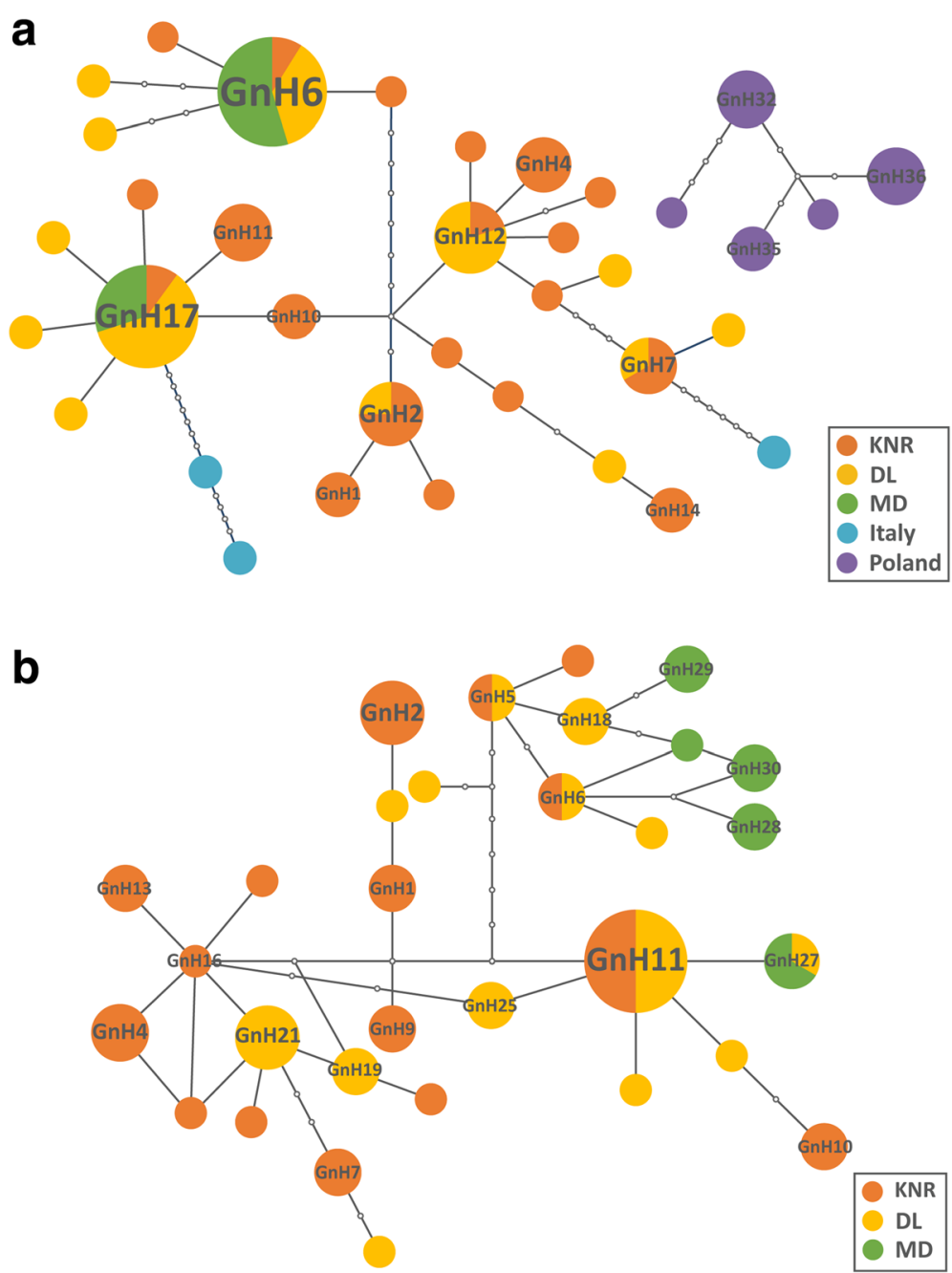

Fig. 3 Haplotype networks for G. nasalis. a Haplotype network for G. nasalis based on the cox1 gene. b Haplotype network for G. nasalis based on the cox2 gene

$\mathrm{MD}$ ) and 0.024 (DL and $\mathrm{MD}$ ), whereas distances based on cox2 gene sequences were 0.006 (KNR and DL), 0.015 (KNR and MD) and 0.017 (DL and MD). These results demonstrate that the genetic distance was smallest between KNR and DL populations and larger than for other populations. Fst values for cox 1 sequences were 0.03245 (KNR and DL), 0.19067 (KNR and MD) and 0.22841 (DL and MD), while the corresponding $\mathrm{Nm}$ values were $7.45416,1.06116$ and 0.84452 , respectively. Fst values for cox 2 sequences were 0.04216 (KNR and DL), 0.17652 (KNR and MD) and 0.17893 (DL and MD), and $\mathrm{Nm}$ values were $5.67979,1.16627$ and 1.14719 , respectively. These results indicate that there was a low degree of genetic differentiation ( Fst $<0.05, \mathrm{Nm}>4$ ) between KNR and DL populations, and moderate genetic differentiation $(0.15<F s t$ $<0.25)$ and low level of gene flow $(\mathrm{Nm}<4)$ between MD and the other two populations.

\section{Gasterophilus intestinalis}

Genetic distances among different populations based on cox 1 sequences are shown in Table 1. Mean genetic distances among Chinese, Italian and Polish populations ranged between $0.018-0.023$. The distances among populations in China did not differ significantly (0.0090.012), whereas the distance between KNR and DL populations based on cox 2 sequences was 0.006 . Fst and $\mathrm{Nm}$ values for $\operatorname{cox} 1$ sequences among the six populations are shown in Table 2. Considerable genetic differentiation $(F s t>0.25)$ and low level of gene flow $(\mathrm{Nm}<4)$ occur among Italian, Polish and Chinese populations, but restricted by the very small sample size of Italian population, this result remains to be verified. Genetic differentiation was low among KNR, DL and DQ populations (Fst $<0.05, N m>4)$ and moderate among the remaining populations in China $(0.05<F s t<0.15)$, 

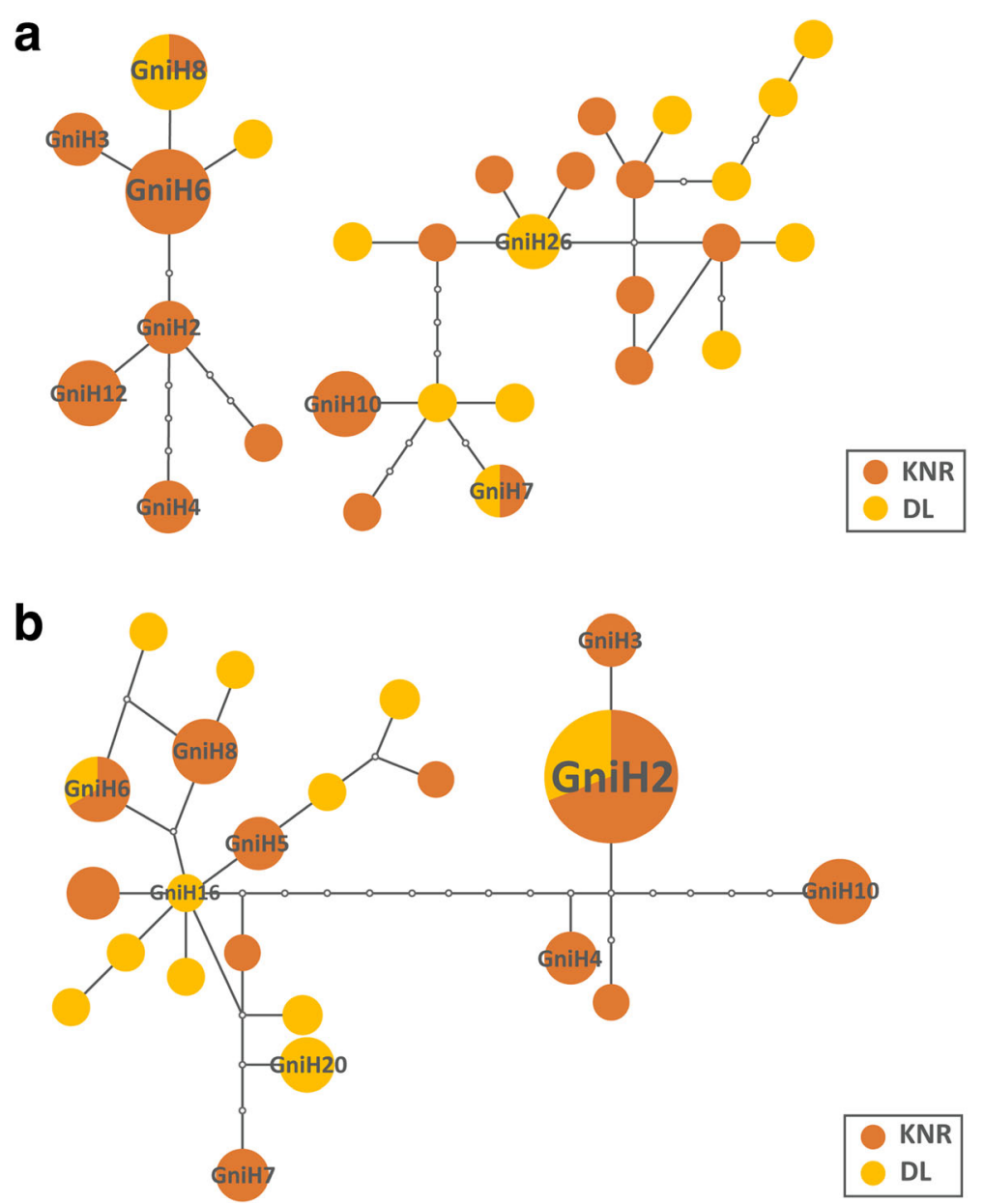

Fig. 4 Haplotype networks for G. nigricornis. a Haplotype network for G. nigricornis based on the cox1 gene. b Haplotype network for G. nigricornis based on the cox2 gene

which also exhibited a low degree of gene flow $(\mathrm{Nm}<4)$. Fst and $\mathrm{Nm}$ values for cox 2 sequences between KNR and DL were 0.01847 and 13.28546, respectively, indicating a low level of genetic differentiation $(F s t<0.05)$ and frequent gene flow $(\mathrm{Nm}>4)$.

\section{Gasterophilus nasalis}

Mean genetic distances among populations based on cox 1 sequences are shown in Table 3. Distances based on cox 2 sequences were 0.009 (KNR and DL), 0.015 (KNR and MD) and 0.013 (DL and MD), and those based on $\operatorname{cox} 1$ sequences ranged between $0.009-0.023$, with the closest genetic distance observed between KNR and DL populations. The sequence dataset for cox 2 also revealed a close genetic distance between KNR and DL and showed that MD was distant from the other populations. Fst and $N m$ values for the cox 1 gene among the four populations are shown in Table 4. Apart from the low degree of genetic differentiation $(F s t<0.05)$ and frequent gene flow $(\mathrm{Nm}>4)$ between KNR and DL, the other populations showed high genetic differentiation $($ Fst $>0.25)$ and low gene flow $(\mathrm{Nm}<4)$.

\section{Gasterophilus nigricornis}

The mean genetic distances between KNR and DL populations calculated based on $\operatorname{cox} 1$ and $\operatorname{cox} 2$ sequences were 0.019 and 0.013 , respectively. Fst and $\mathrm{Nm}$ values for the cox 1 gene between KNR and DL were 0.07371 and 3.14167 , respectively, while those for the cox 2 gene were 0.08215 and 2.79321 , respectively. These results showed that there was little genetic differentiation $(F s t>0.05)$ and low level of gene flow $(\mathrm{Nm}<4)$ between KNR and DL.

\section{Demographic history and migration}

Populations are often far from equilibrium, and not all deviate in the same direction. The neutrality of all populations 


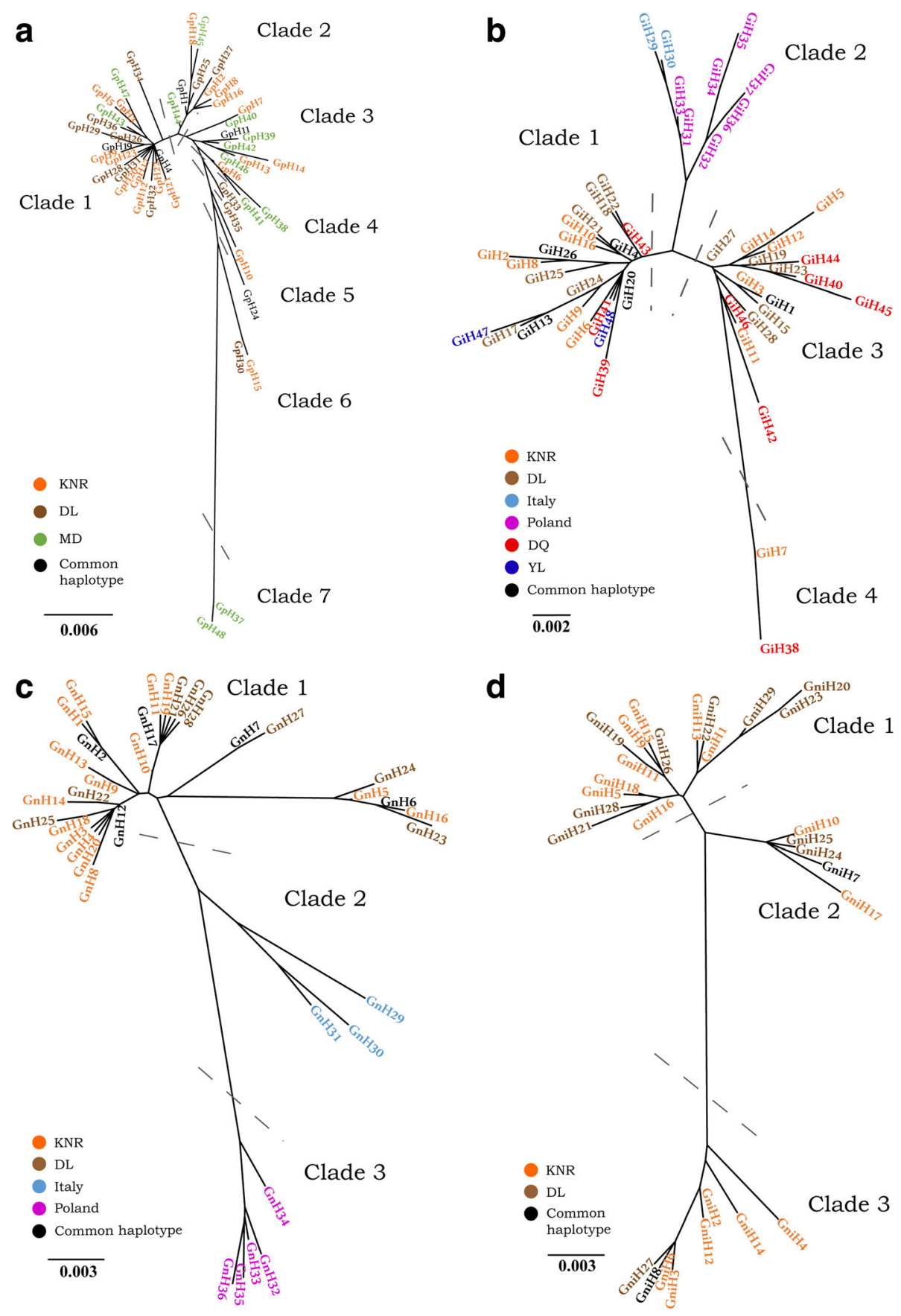

Fig. 5 Neighbor-joining tree based on the cox1 gene for G. pecorum, G. intestinalis, G. nasalis and G. nigricornis. a Gasterophilus pecorum. b Gasterophilus intestinalis. c Gasterophilus nasalis. d Gasterophilus nigricornis

was estimated using Tajima's $D$ and Fu's $F s$ (Additional file 2: Table S3). The results of the neutrality test indicated a recent rapid expansion in two populations (KNR and DL) of G. pecorum, three populations (KNR, DL and DQ) of G. intestinalis, and in the KNR population of G. nasalis, whereas the other populations had remained relatively stable.

The LAMARC analysis (Additional file 2: Table S4) revealed that population size $(\theta)$ and exponential growth rate (g) were relatively high for the DL population followed by the KNR population of G. pecorum, while the low $\theta$ value and negative $g$ value of the MD population indicated a declining trend. Migration was high from DL to KNR and from KNR to MD, whereas the emigration and immigration rates of $\mathrm{MD}$ and $\mathrm{DL}$, respectively, were low. Among G. intestinalis populations, DQ had the highest $\theta$ and $g$ values followed by KNR. High migration was observed among 

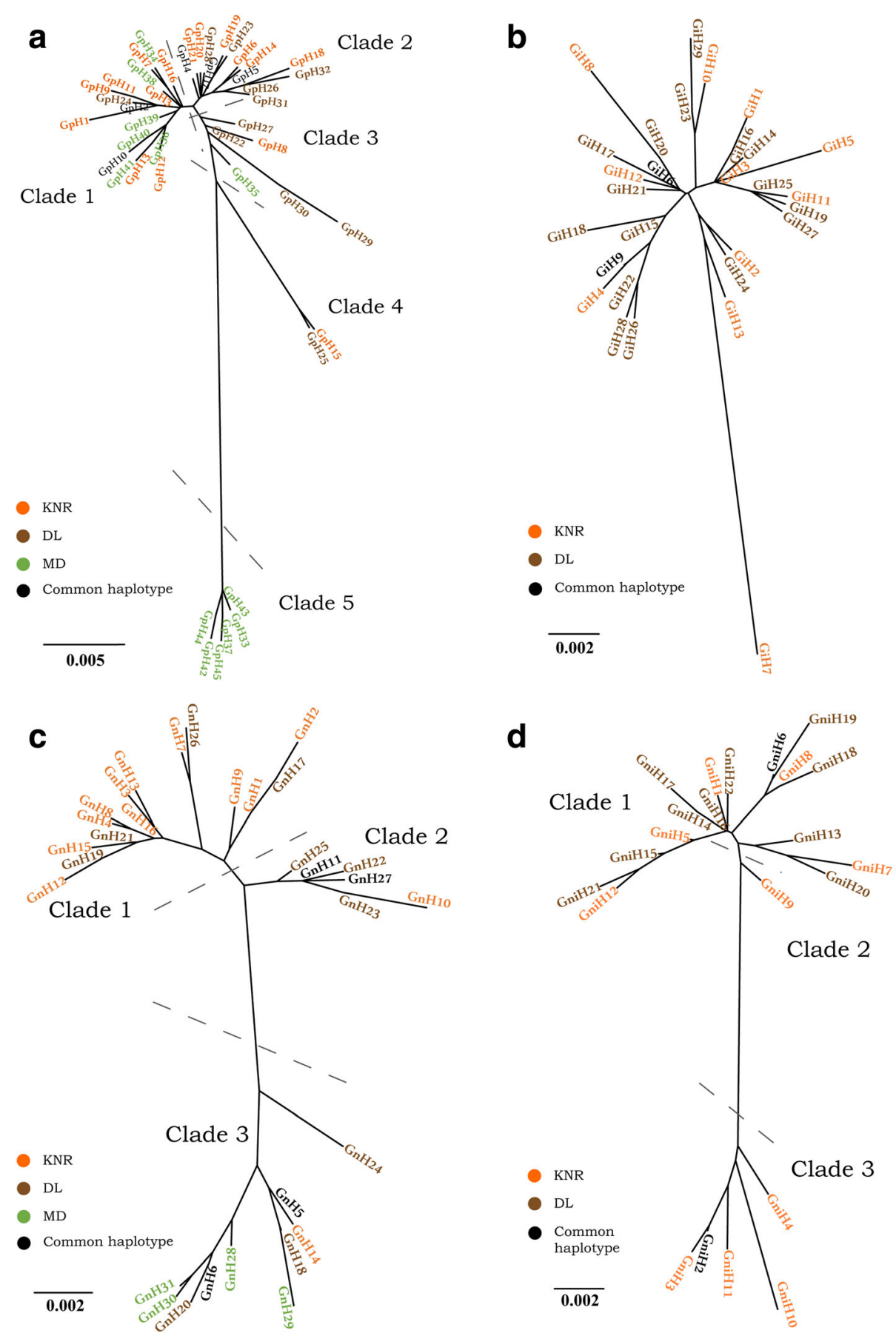

Fig. 6 Neighbor-joining tree based on the COll gene for G. pecorum, G. intestinalis, G. nasalis and G. nigricornis. a Gasterophilus pecorum. b Gasterophilus intestinalis. c Gasterophilus nasalis. d Gasterophilus nigricornis

Chinese populations, including from DL to KNR, DL to $\mathrm{YL}, \mathrm{DL}$ to $\mathrm{DQ}$ and $\mathrm{YL}$ to $\mathrm{DQ}$. In general, KNR populations had low emigration and high immigration rates, while the opposite was true for DL. In European populations, migration was more frequent from Poland to Italy than in the other direction. The relationship between the Italian and Chinese populations was more complex than that between the Polish and Chinese populations, but the results may be biased due to the limited number of samples from Italy. Among G. nasalis, the highest $g$ and $\theta$ values were observed in the MD and Italy populations, respectively. In China, the highest rates of migration were from DL to KNR and MD. The migration rates from Italy to Poland were even higher, while those between the Chinese and European populations 
Table 1 Mean genetic distance between G. intestinalis populations in distinct areas based on mitochondrial cytochrome $c$ oxidase subunit 1 gene

\begin{tabular}{llllll}
\hline Population & KNR & DL & Italy & Poland & DQ \\
\hline DL & 0.009 & - & - & - & - \\
Italy & 0.018 & 0.015 & - & - & - \\
Poland & 0.014 & 0.013 & 0.009 & - & - \\
DQ & 0.012 & 0.010 & 0.019 & 0.016 & - \\
YL & 0.010 & 0.007 & 0.014 & 0.013 & 0.012 \\
\hline
\end{tabular}

were generally low. The $\theta$ values of $G$. nigricornis in KNR and Dowered were similar, whereas $g$ values were higher in DL than in KNR. Migration rates between the two populations were similarly high, with migration from KNR to DL being slightly higher.

\section{Discussion}

In this study, analyses of the mitochondrial cox 1 and cox2 genes of G. pecorum, G. intestinalis, G. nasalis and $G$. nigricornis revealed that besides $\operatorname{cox} 1$ gene haplotypes in the DL population of G. nasalis, the other populations of each species showed high $H d$, indicating a high degree of cox 1 and cox 2 gene polymorphism in Gasterophilus species, all of which showed a high degree of concordance with fewer common haplotypes and fewer samples sharing the same haplotype. The high diversity of $G$. pecorum has been previously reported [35]. Compared to the mitochondrial $\operatorname{cox} 1$ gene, the $\operatorname{cox} 2$ sequences of G. pecorum and G. nasalis showed higher $H d$ and $\pi$ and more private haplotypes. The haplotype networks and phylogenetic trees revealed that haplotypes of $\mathrm{DL}$ and MD populations of G. pecorum had distinct distributions that were similar to the trends of G. nasalis. The Italian, Polish and Chinese populations of G. intestinalis clustered into three clades, all Chinese isolates clustered together without geographical separation. Compared to the G. nigricornis KNR population, MD showed a certain degree of aggregation.

Host migration affects the genetic structure of parasite populations [36, 37]. The geographical distribution of species is closely related to biological and non-biological

Table 2 Pairwise fixation index (Fst: below diagonal) and gene flow (Nm: above diagonal) values for six G. intestinalis populations

\begin{tabular}{lllllll}
\hline Population & KNR & DL & Italy & Poland & DQ & YL \\
\hline KNR & - & 9.42867 & 0.14350 & 0.32216 & -12.92101 & 1.88931 \\
DL & 0.02583 & - & 0.10156 & 0.22386 & 13.38884 & 2.84521 \\
Italy & 0.63532 & 0.71112 & - & 0.18810 & 0.17816 & 0.11111 \\
Poland & 0.43694 & 0.52758 & 0.57065 & - & 0.39733 & 0.20365 \\
DQ & -0.01973 & 0.01833 & 0.58389 & 0.38620 & - & 1.70542 \\
YL & 0.11686 & 0.08077 & 0.69231 & 0.55109 & 0.12785 & - \\
\hline
\end{tabular}

Table 3 Mean genetic distance between G. nasalis populations in distinct areas based on mitochondrial cytochrome $c$ oxidase subunit 1 gene

\begin{tabular}{lllll}
\hline Population & KNR & DL & MD & Italy \\
\hline DL & 0.009 & - & - & - \\
MD & 0.012 & 0.011 & - & - \\
Italy & 0.020 & 0.021 & 0.023 & - \\
Poland & 0.018 & 0.019 & 0.023 & 0.017 \\
\hline
\end{tabular}

factors in the ecological niche, including exogenous factors such as geological events, changes in climate and food distribution as well as intrinsic species characteristics such as behavioral sensitivity, food web specificity and diffusibility. These factors act either directly or indirectly on both the host and parasite, with more profound effects on the latter [38]. The life-cycle of Gasterophilus spp. consists of the obligate parasitic larval stage on the horse and the free-living adult stage, and is influenced by host movement and environment. The three sampling sites in China selected for this study represent different types of grassland with distinct climates. Only G. pecorum and G. nasalis were collected in $\mathrm{MD}$, our analysis revealed a moderate degree of genetic differentiation and a lack of gene flow between MD and populations in other regions. In contrast, more frequent genetic flow was observed between the KNR and DL populations of all four species. In general, the four Gasterophilus species showed a consistent population genetic structure in the KNR and DL populations, whereas two of the species in MD differed from their counterparts in KNR and DL. This implies that even if there is host exchange as a part of livestock husbandry trade, the natural conditions of the Qinghai-Tibet Plateau characterized by an alpine climate and high altitude differ from those of the other two regions located on natural geographical barriers such as Kunlun and Qilian Mountains, which can lead to genetic differentiation while preventing gene exchange among populations. Xinjiang and Inner Mongolia constitute a single zoogeographical region (Mongo-Xinjiang region) [24, 25]; consistent with this, our results suggest that Gasterophilus populations in KNR of Xinjiang and DL of Inner Mongolia share similar a genetic structure and species type.

Table 4 Pairwise fixation index (Fst: below diagonal) and gene flow (Nm: above diagonal) values for five $G$. nasalis populations

\begin{tabular}{llllll}
\hline Population & KNR & DL & MD & Italy & Poland \\
\hline KNR & - & 9.00583 & 0.51422 & 0.26556 & 0.16560 \\
DL & 0.02701 & - & 1.27588 & 0.29397 & 0.17310 \\
MD & 0.32713 & 0.16384 & - & 0.20223 & 0.11048 \\
Italy & 0.48491 & 0.45958 & 0.55282 & - & 0.29553 \\
Poland & 0.60015 & 0.59088 & 0.69352 & 0.45827 & - \\
\hline
\end{tabular}


Gasterophilus pecorum, G. intestinalis and G. nasalis from DL showed a consistently high migration rate, suggesting that DL is the origin of Gasterophilus flies in Mongolia-Xinjiang and Qinghai-Tibet regions of China. The Xinjiang Uygur Autonomous Region includes KNR at the heart of the Eurasian continent. Historically, the Silk Road of China passed through this area, which saw frequent trade of animals such as horses that were important means of transportation [39]. The generally higher migration rates of Gasterophilus flies in KNR indicate that Gasterophilus spp. in the area spread from multiple locations, likely reflecting the frequent movement of their hosts. Although we sampled a limited number of sites in this study, our results nonetheless demonstrate that Gasterophilus spp. migrated westward from the grasslands of eastern China. The neutrality test results showed that G. pecorum and G. intestinalis underwent significant population expansion and were the predominant species in KNR and DL, respectively.

\section{Conclusions}

We investigated the genetic structure and inter-population relationships of four common Gasterophilus species of different geographical populations based on analyses of mitochondrial $\operatorname{cox} 1$ and $\operatorname{cox} 2$ genes. Based on these results, we propose dispersal patterns of these species in China's three major pastoral areas.

\section{Additional files}

Additional file 1: Figure S1. Map of sampling sites in China. (PDF $5098 \mathrm{~kb}$ ) Additional file 2: Table S1. Number of individuals (n), haplotypes, number of haplotypes (denoted in parentheses), haplotype diversity $(\mathrm{Hd}$ ), and nucleotide diversity $(\pi)$ based on the mitochondrial cytochrome $c$ oxidase subunit 1 gene in G. pecorum, G. intestinalis, G. nasalis and G. nigricornis. Table S2. Number of individuals ( $n$ ), haplotypes, number of haplotypes (denoted in parentheses), haplotype diversity $(\mathrm{Hd})$, and nucleotide diversity $(\pi)$ based on the mitochondrial cytochrome $c$ oxidase subunit 2 gene in G. pecorum, G. intestinalis, G. nasalis and G. nigricornis. Table S3. Neutrality test for G. pecorum, G. intestinalis, $G$. nasalis and G. nigricornis in different geographical locations. Table S4. Maximum likelihood estimates of population size $(\theta)$, exponential growth rate $(g)$, and migration rate for different populations of G. pecorum, $G$. intestinalis, G. nasalis and G. nigricornis. (DOCX $27 \mathrm{~kb}$ )

\footnotetext{
Abbreviations

cox1: Mitochondrial cytochrome c oxidase subunit 1 gene; cox2: Mitochondrial cytochrome c oxidase subunit 2 gene; DL: Duolun County, Inner Mongolia Autonomous Region; DQ: Daqing City, Heilongjiang Province; Fst: Pairwise fixation index; g: Exponential growth rate; Hd: Haplotype diversity; KNR: Kalamaili Nature Reserve, Xinjiang Uigur Autonomous Region; LAMARC: Likelihood Analysis with Metropolis Algorithm using Random Coalescence; MD: Maduo County, Qinghai Province; NJ: Neighbor-joining; Nm: Gene flow; YL: Yili Kazak Autonomous Prefecture, Xinjiang Uigur Autonomous Region; $\theta$ : Population size; $\pi$ : Nucleotide diversity
}

\section{Acknowledgements}

We would like to thank the Xinjiang Kalamaili Mountain Nature Reserve for providing all the samples used in this study and for valuable technical assistance.

\section{Funding}

This work was supported by the National Science Foundation of China (No. 31670538), the Fundamental Research Funds for the Central Universities (No. JC2015-04), the Project of Department for Wildlife and Forest Plants Protection, SFA of China (No. 2015-123), and the Student's Platform for Innovation and Entrepreneurship Training Program (No. 201610022073). The funders had no role in the study design, data collection and analysis, decision to publish, or preparation of the manuscript

\section{Availability of data and materials}

The data supporting the conclusions of this article are included within the article and its additional files. The sequence datasets generated during this study have been made available in the GenBank database under the accession numbers MG815141-MG815634.

\section{Authors' contributions}

$\mathrm{BZ}$ and $\mathrm{KL}$ conceived the experiments. $\mathrm{BZ}$ and $\mathrm{HH}$ undertook sampling work. $B Z, H W, H C, X M, Y G$ and $M E$ conducted the experiments. BZ, HH, DZ and $\mathrm{KL}$ analyzed the results. BZ wrote the manuscript. All authors read and approved the final manuscript.

\section{Ethics approval}

The study was performed in accordance with the relevant guidelines and regulations regarding animal welfare. All experimental protocols were approved by Wildlife Conservation Office of Altay Prefecture and Beijing Forestry University.

\section{Consent for publication}

Not applicable.

\section{Competing interests}

The authors declare that they have no competing interests.

\section{Publisher's Note}

Springer Nature remains neutral with regard to jurisdictional claims in published maps and institutional affiliations.

\section{Author details}

${ }^{1}$ Key Laboratory of Non-Invasive Research Technology for Endangered Species, College of Nature Conservation, Beijing Forestry University, Beijing 100083, China. ${ }^{2}$ Qinhuangdao Forestry Bureau, Qinhuangdao 066004, Hebei, China. ${ }^{3}$ Wildlife Conservation Office of Altay Prefecture, Altay 836599, Xinjiang, China. ${ }^{4}$ Xinjiang Research Centre for Breeding Przewalski's Horse, Urumqi 831700, Xinjiang, China.

Received: 3 March 2018 Accepted: 30 July 2018

Published online: 22 August 2018

\section{References}

1. Zumpt F. Myiasis in man and animals in the old world: a textbook for physicians, veterinarians and zoologists. London: Butterworths; 1965.

2. Fan ZD. Index of common flies in China. Beijing: Science Press; 1992.

3. Wang MF. Flies in China (Vol. II). Shenyang: Science and Technology Press: 1998

4. Li K, Wu Z, Hu DF, Cao J, Wang C. A report on new causative agent (Gasterophilus spp.) of the myiasis of Przewalski's horse occurred in China. Acta Vet Et Zootech Sin. 2007;38:837-40.

5. Hall M, Wall R. Myiasis of humans and domestic animals. Adv Parasitol. 1995. 35:257-334

6. Sequeira JL, Tostes RA, Oliveira-Sequeira TCG. Prevalence and macro and microscopic lesions produced by Gasterophilus nasalis (Diptera: Oestridae) in the Botucatu Region, SP, Brazil. Vet Parasitol. 2001;102:261-6.

7. Sánchez-Andrade R, Cortiñas FJ, Francisco I, Sáncheza JA, Mulab P., Cazapal $C$, et al. A novel second instar Gasterophilus excretory/secretory antigenbased ELISA for the diagnosis of gasterophilosis in grazing horses. Vet Parasitol. 2010;171:314-20. 
8. Edwards GT. The prevalence of Gasterophilus intestinalis in horses in northern England and Wales. Vet Parasitol. 1982;11:215-22.

9. Hatch C, McCaughey WJ, O'Brien JJ. The prevalence of Gasterophilus intestinalis and $G$ nasalis in horses in Ireland. Vet Rec. 1976;98:274-6.

10. Lyons ET, Swerczek TW, Tolliver SC, Bair HD, Drudge JH, Ennis LE. Prevalence of selected species of internal parasites in equids at necropsy in central Kentucky (1995-1999). Vet Parasitol. 2000;92:51-62.

11. Otranto D, Milillo P, Capelli G, Colwell DD. Species composition of Gasterophilus spp. (Diptera, Oestridae) causing equine gastric myiasis in southern Italy: parasite biodiversity and risks for extinction. Vet Parasitol. 2005;133:111-8.

12. Niedźwiedź A, Borowicz H, Nicpoń JM. Prevalence study in horses infected by Gasterophilus sp. in an eastern region of Poland. Vet Parasitol. 2013;191:94-6.

13. Pilo C, Altea A, Scala A. Gasterophilosis in horses in Sardinia (Italy): effect of meteorological variables on adult egg-laying activity and presence of larvae in the digestive tract, and update of species. Parasitol Res. 2015;114:1693-702.

14. Wang W, Xiao S, Huang H, Li K, Zhang D, Chu H, et al. Diversity and infection of Gasterophilus spp. in Mongol- Xinjiang Region and Qinghai Tibet Region. Sci Silvae Sin. 2016;52:134-9.

15. Fresia P, Azeredo-Espin AML, Lyra ML. The phylogeographic history of the new world screwworm fly, inferred by approximate Bayesian computation analysis. PLoS One. 2013;8:e76168.

16. Ståhls G, Vujić A, Petanidou T, Cardoso P, Radenković S, Ačanski J, et al. Phylogeographic patterns of Merodon hoverflies in the Eastern Mediterranean region: revealing connections and barriers. Ecol Evol. 2016;6: 2226-45.

17. Dincă V, Runquist M, Nilsson M, Vila R. Dispersal, fragmentation, and isolation shape the phylogeography of the European lineages of Polyommatus (Agrodiaetus) ripartii (Lepidoptera: Lycaenidae). Biol J Linn Soc. 2013;109:817-29.

18. Miranda EA, Batalha-Filho H, Congrains C, Carvalho AF, Ferreira KM, Del Lama MA. Phylogeography of Partamona rustica (Hymenoptera, Apidae), an endemic stingless bee from the Neotropical Dry Forest Diagonal. PLoS One. 2016:11:e0164441.

19. Ye Z, Zhu G, Damgaard J, Chen X, Chen P, Bu W. Phylogeography of a semi-aquatic bug, Microvelia horvathi (Hemiptera: Veliidae): an evaluation of historical, geographical and ecological factors. Sci Rep. 2016;6:21932.

20. Pawlas-Opiela M, Wojciech $Ł$, Sołtysiak Z, Otranto D, Ugorski M. Molecular comparison of Gasterophilus intestinalis and Gasterophilus nasalis from two distinct areas of Poland and Italy based on cox 1 sequence analysis. Vet Parasitol. 2010;169:219-21.

21. Gao DZ, Liu GH, Wang GL, Zhu XQ, Wang CR. Sequence variation in three mitochondrial genes among Gasterophilus intestinalis isolates from two distinct regions in China. Mitochondrial DNA A DNA Mapp Seq Anal. 2017; 28:37-40.

22. Chu HJ, Jiang ZG, Ge Y, Jiang F, Tao Y, Wang C. Population densities and number of khulan and goitred gazelle in Mt. Kalamaili Ungulate Nature Reserve. Biodiversity Sci. 2009;17:414-22.

23. Aruhan YC. Analysis on the characteristics of climate change in recent 50 years in Duolun County, Inner Mongolia. J Inner Mongolia Univ. 2007;38:434-8.

24. Cheng TH, Chang YT. On tentative scheme for dividing zoogeographical regions of China. Acta Geogr Sin. 1956;1:2.

25. Zhang RZ. Zoogeography of China. Beijing: Science Press; 1999.

26. Han Y, Li G. Climate change of Yellow River source region for nearly 30 years in Maduo County. J Qinghai Meteor. 2011;2:36-41.

27. Sambrook J, Russell DW. Molecular Cloning: A Laboratory Manual. 3rd ed. New York: Cold Spring Harbor Laboratory Press; 2001.

28. Simon C, Frati F, Beckenbach A, Crespi B, Liu H, Flook P. Evolution, weighting, and phylogenetic utility of mitochondrial gene sequences and a compilation of conserved polymerase chain reaction primers. Ann Entomol Soc Am. 1994;87:651-701.

29. Hall TA. BioEdit: a user-friendly biological sequence alignment editor and analysis program for Windows 95/98/NT. Nucleic Acids Symp Ser. 1999:41:95-8.

30. Kumar S, Stecher G, Tamura K. MEGA7: Molecular Evolutionary Genetics Analysis version 7.0 for bigger datasets. Mol Biol Evol. 2016;33:1870-4.

31. Librado P, Rozas J. DnaSP v5: a software for comprehensive analysis of DNA polymorphism data. Bioinformatics. 2009;25:1451-2.

32. Felsenstein J. Confidence limits on phylogenies: an approach using the bootstrap. Evolution. 1985;39:783-91.

33. Clement M, Posada D, Crandall KA. TCS: a computer program to estimate gene genealogies. Mol Ecol. 2000;9:1657-9.
34. Kuhner MK. LAMARC 2.0: maximum likelihood and Bayesian estimation of population parameters. Bioinformatics. 2006;22:768-70.

35. Wang W, Zhang D, Hu D, Chu H, Cao J, Ente M, et al. Population genetic structure of Gasterophilus pecorum in the Kalamaili Nature Reserve, Xinjiang, based on mitochondrial cytochrome oxidase (COI) gene sequence. Med Vet Entomol. 2014;28:75-82.

36. Morgan ER, Clare EL, Jefferies R, Stevens JR. Parasite epidemiology in a changing world: can molecular phylogeography help us tell the wood from the trees? Parasitology. 2012;139:1924-38.

37. McCoy K, Beis P, Barbosa A, Cuervo JJ, Fraser WR, González-Solís J, et al. Population genetic structure and colonisation of the western Antarctic Peninsula by the seabird tick /xodes uriae. Mar Eco Prog Ser. 2012;459:109-20.

38. Criscione CD, Poulin R, Blouin MS. Molecular ecology of parasites: elucidating ecological and microevolutionary processes. Mol Ecol. 2005;14:2247-57.

39. Liu X. The Silk Road in world history. New York: Oxford University Press; 2010.
Ready to submit your research? Choose BMC and benefit from:

- fast, convenient online submission

- thorough peer review by experienced researchers in your field

- rapid publication on acceptance

- support for research data, including large and complex data types

- gold Open Access which fosters wider collaboration and increased citations

- maximum visibility for your research: over $100 \mathrm{M}$ website views per year

At BMC, research is always in progress.

Learn more biomedcentral.com/submissions 